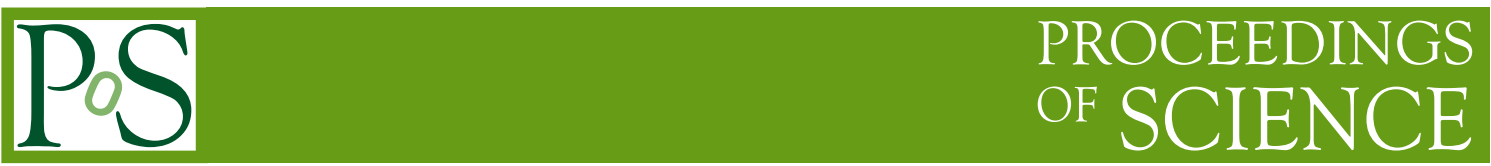

\title{
A GPU based Transient Dedisersion Search Engine for CRAFT
}

Richard Dodson*,Christopher Harris, Sabyasachi Pal, and Randall Wayth, Int. Centre for Radio Ast. Research (ICRAR), Perth, Australia

E-mail: richard.dodsoneicrar.org

The Commensal Realtime ASKAP Fast Transient Survey (CRAFT[5]) will search the ASKAP data stream for fast $(<5 \mathrm{sec})$ transient events, associated with the most extreme conditions in the Universe. The CRAFT search will run in parallel with all normal observing on ASKAP, giving a tremendous advance in the transient parameter space which can be searched. ASKAP offers high sensitivity, high resolution, and continous observations of a significant portion of the sky. However, to search across the wide field of view and at the data rates which are being provided by ASKAP is extremely challenging. Nevertheless, as reported here, we are on track to achieve the goals as laid out in the Survey Science Proposal. Using GPUs as a simple highly parallel compute-engine we can monitor the full field of view with a $5 \sigma$ sensitivity of $\sim \mathrm{Jy}$ for a millisecond event covering the astronomically significant range of DMs. After that trigger detection we can download the beamformer data-buffer and image the sky at full sensitivity and spatial resolution with an arbitary frequency and time resolution.

ISKAF2010 Science Meeting - ISKAF2010

June 10-14, 2010

Assen, the Netherlands

* Speaker. 


\section{The CRAFT Survey}

The frontiers of physics are advanced by observations of the extremes, and short-timescale Dynamic Sources are associated with the most extreme energy density events in the Universe. They can be used as the ultimate laboratory; their emission must be generated by states of matter that probe physical regimes that far exceed the range achievable in any terrestrial experiment. The existence alone of such high energy densities transforms our understanding of the behaviour of matter and space-time under these conditions. The Commensal Realtime ASKAP Fast Transient (CRAFT) Science Survey Project will capture these events using ASKAP. Both the survey and the instrument will extend Radio Astronomy into high time resolution single-pulse observations that will continuously monitor a significant portion of the sky.

The traditional operational mode of most radio telescope precluded the possibility of detecting rare sources with short lifetimes. Up till now the most sensitive radio telescopes, by design, have small fields of view, and positional accuracy comes from combining long observations, with a number of antennas in an array, hiding all short timescale events. As expounded in a number of places (e.g. [2]) there is the realisation that one of the unexplored regions of 'parameter space' that the SKA will allow us to explore is that of short timescale transient sources.

A major new facility that will open this area is ASKAP, which is being built in Western Australia by CSIRO. The prototype is expected to be functioning by 2011, and the full array by 2013. ASKAP is formed from 36 antennas each with a 30 sq. deg. Field of View (FoV). Therefore it is ideal for the search for Highly Dynamic Sources with high sensitivity and with sufficient sky coverage to catch rare events.

There are a number of existing pre-SKA surveys to hunt for such events, such as the ALFA transient survey [4], the High Time Resolution Survey [7] on the Parkes-Multibeam and the Flyseye survey [10] with the ATA dishes. These are indicated on Figure 1, which shows the resolution (in degrees) and sky-coverage (in degrees ${ }^{2}$ ) against sensitivity. To normalise (and simplify) this figure all surveys have been assumed to have a common bandwidth and system temperature of $300 \mathrm{MHz}$ and $25 \mathrm{~K}$, so that the vertical axis is in Jys. The three mentioned surveys are essentially single-dish surveys improved by a 'multi-view' scale factor. This is provided by the 13-beams in one dish for the Parkes-MB survey or the 42-dishes pointed in different directions for the Flys-Eye survey. These surveys therefore only probe the 'explored' zone between the green line (single dish resolution) and the red line (multi-view FoV) in Figure 1. The CRAFT survey on the other hand, being an interferometer with a wide FoV, has a good sensitivity to rare events combined with high positional accuracy and spans $30 \mathrm{sq}$. deg. with a post-detection resolution of $10^{\prime \prime}$, as indicated by the gray region.

\section{The Targets}

Predictions have been made of a number of the types of events that would measure fundamental astronomical parameters, and in the last few years there have been some detections of these classes of signals. These are well reported elsewhere, so here we only present a summary. 


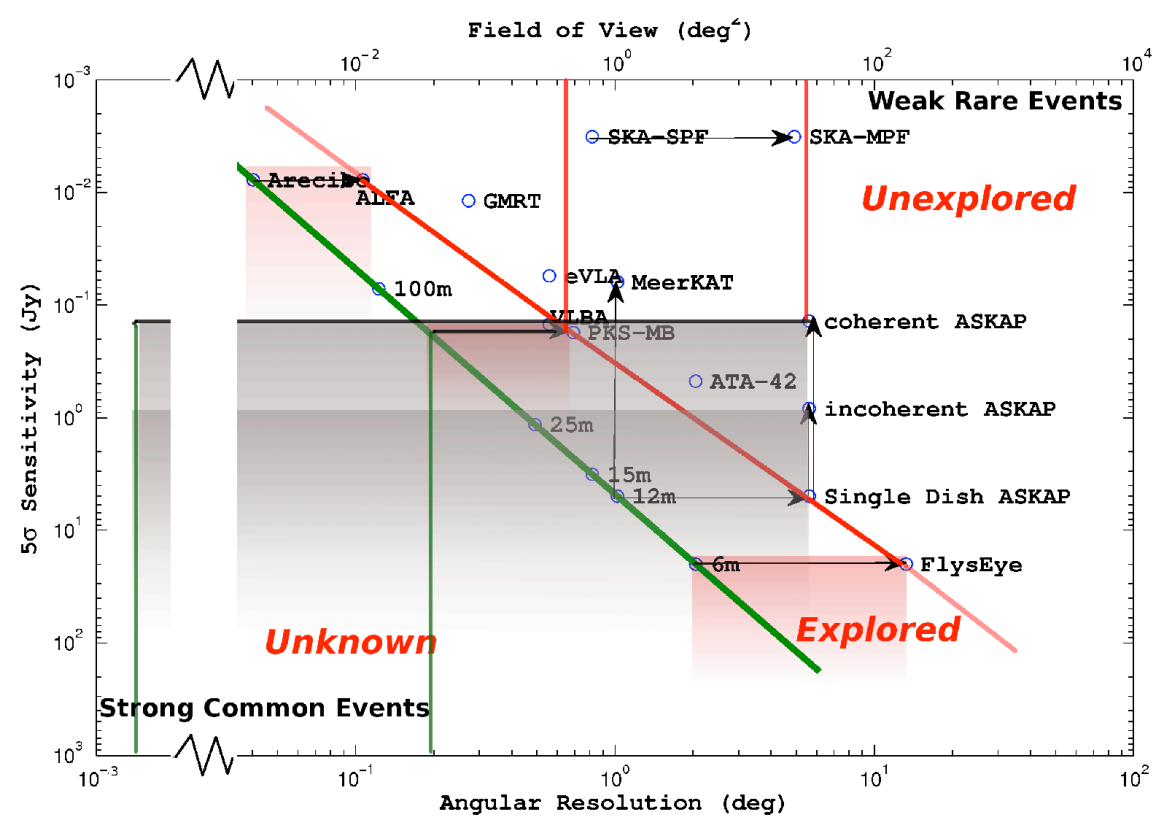

Figure 1: The CRAFT survey will search a greater area of parameter space than other similar surveys. The parameter space searched by the Arecibo-ALFA, Parkes-MB and ATA-FlysEye surveys are indicated with orange boxes. These are bound between the green line, the single dish resolution and the red line, the FoV with a multi-beam instrument. CRAFT has wide FoV and high resolution as indicated by the gray area. This is an essential pre-requiste for the identification of a transient source.

The transient events that CRAFT is sensitive to include: pulsar Giant Pulses, which have been observed with brightness temperatures of $10^{37} \mathrm{~K}[6]$. We would be able to detect such a pulse across our Galaxy and beyond.

Rotating RAdio Transient Sources (RRATS)[9] are similar to normal pulsars but with very low duty cycles, having detectable emission for less than a second per day. To make a full survey of RRATS populations we require single-pulse searches with wide FoV instruments. As such, the CRAFT survey is well matched to this task.

Similarly, the Magnetar J1810-197 has been detected with peak single-pulse amplitudes $\sim 10$ Jy and pulse widths $\sim 0.15 \mathrm{~s}[1]$. Such sources could be detected across the Galaxy by CRAFT, potentially converting ASKAP into the premier Magnetar detector.

CRAFT has been developed with the goal of discovering more examples of the mysterious 'Lorimer Burst'[8]. As such we have developed a system which will provide high time resolution single-pulse observations that will continuously monitor a significant portion of the sky, which is essential to find more of this clearly rare class of sources. Several approaches are being tried. We present in this paper the GPU-based Transient Dedispersion Search Engine which is able to detect transients in real-time. This is followed up by the offline analysis of a data-buffer and therefore provide the dispersion, time and most importantly the position of the transient event. Full details 
can be found on the CRAFT webpages ${ }^{1}$.

The ASKAP beamformer stores the data in memory in a circular data buffer and also forms the autocorrelations which it provides to the CRAFT external hardware for an incoherent search. These data are sampled at every millisecond and every $\mathrm{MHz}$ and will be transmitted directly to the system as UDP data-packets. Once this system detects a transient event a trigger is passed back to the beamformer and the stored data is downloaded from memory for complete, full sensitivity, offline analysis.

\section{The CRAFT GPU Dedispersion Search Engine}

The CRAFT first phase solution for the transient detection is to search in the summed total powers (per beam) from all antennas for event triggers. On the detection of an event, and the passing of several simple sanity checks, the data buffer is downloaded for full, offline, imaging.

The ASKAP beamformer will be used to provide the autocorrelations, for each $1 \mathrm{MHz}$ channel, for each beam, for each millisecond. This gives out 2-bytes words for the power in every one of the $3041 \mathrm{MHz}$-channels and between 16-36 dual-polarisation beams every millisecond, and from every antenna. This quite modest data rate is easily handled by off-the-shelf systems. We wish to search from low Dispersion Measures (DM) of around ten $\mathrm{pc} / \mathrm{cm}^{3}$, which excludes earth-origin pulsed emissions, to values up to three thousand or so, which would in found in pulsed emission which passed directly through the galactic centre. Based on these values a rough operations count gives the required performance as around 50 G-FLOPS. The GPU provides a highly parallel processor, which can repeat multiple operations on single datasets (i.e. multiple dedispersions), in an easily programmed environment. They provide excellent compute-power per dollar and per watt, both significant considerations for the CRAFT project.

The data transformation from Frequency-domain to the DM-domain is being performed on the GPU. We are running CUDA on a NVIDA 9800-GT (a somewhat obsolete system) yet even with this, the approach we have developed would able to incoherently search all beams with full bandwidth, and millisecond time resolution, in real time, with only two GPUs. The Figures 2a and $2 \mathrm{~b}$ show the output from the Frequency-Time domain to the DM-time domain Transform. The first case is the results from searching simulated ASKAP data at 1.4-GHz containing a very strong $1 \mathrm{~ms}$ burst with a DM of $300 \mathrm{pc} / \mathrm{cm}^{3}$; a 'Lorimer-like' event. DMs between 10 and 1600 were searched. The performance is 18 times real-time, so two GPUs would be capable of searching the whole ASKAP FoV. The second case is for real data from Parkes observing the Vela pulsar which has a DM of $69 \mathrm{pc} / \mathrm{cm}^{3}$, with time sampling of $64 \mu \mathrm{sec}$. The variable flux burst every $89 \mathrm{~ms}$ is clearly detected. The single GPU only manages half real time in this case, but a more modern card would easily be able to keep pace.

\section{Towards the Final System}

The dispersion transform is only a single, but important, stage in the CRAFT transient capture system. In addition we have developed plans for:

\footnotetext{
${ }^{1}$ https://pm.atnf.csiro.au/askap/projects/show/sup-craft
} 


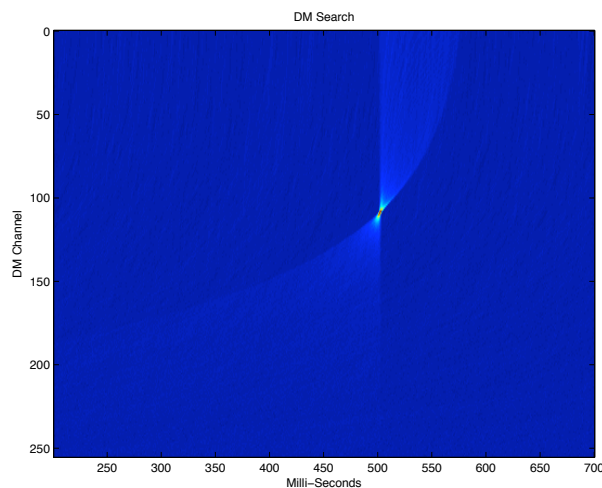

(a) Results from a DM-transform of fake 'Lorimer-ilke' data matching the ASKAP configuration. 5 minutes of single beam data can be processed in 24 seconds, which would allow the processing of 18 beams/GPU in real time.

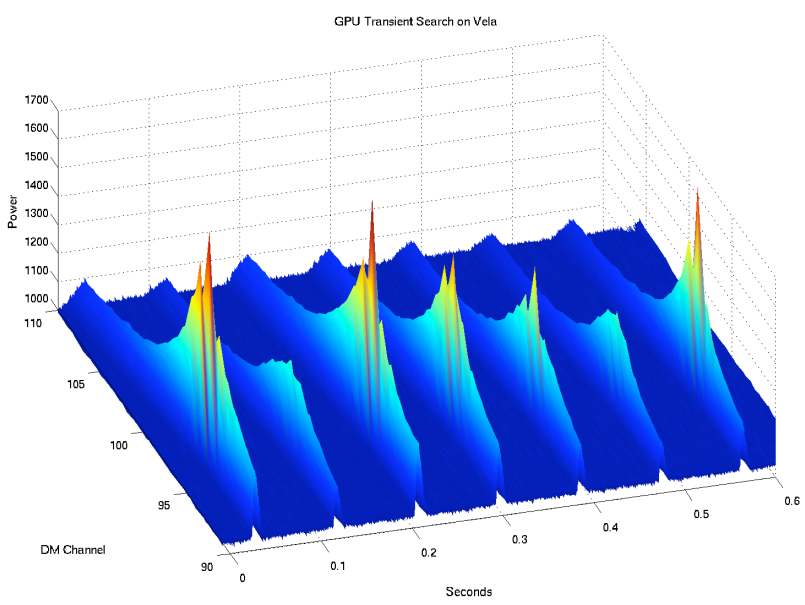

(b) Results from searching data taken from the Parkes High Resolution Timing Survey (1024 channels over 400-MHz with 64 $\mu$ sec resolution) on Vela. The regular single pulses are clearly detected

Figure 2: Results from the GPU de-dispersion system.

- Detection of the Event in the time-DM plane. The initial demonstrator has a simple peak detection, but more sophisticated approaches will be developed.

- Network connection for the data-flow. Collation of the data for the GPU is a significant task, but will be based around the UDP streams from the ASKAP beamformer. A packet format is being defined which will allow multiple machines to collect the data they will need, whilst keeping the number of packets to a minimum and keeping the data rates below network limits.

- The requirements for data-buffers, which are that they must store the raw voltages with sufficient time-spans to recover DMs upto several thousands at an affordable price. We have 
a strategy for the off-loading of the data and for the offline processing. Increasing the onboard beamformer DRAMS to $2 \mathrm{~GB}$ matches most considerations. We plan to offload via the same network as for the trigger, into an offline processing machine which will form the correlations with DiFX [3].

\section{In Conclusion}

We have developed the first phase solution for the CRAFT survey. The GPU system is capable searching the ASKAP beamformer autocorrelation, in real time, and providing the trigger for the download of the baseband data for full sensitivity and resolution analysis of ASKAP detections of fast transient events.

The disadvantages of this approach, which makes an incoherent sum across the frequency channels, is that there is significant sensitivity loss. However to form a coherent sum would require one search per synthesised beam rather than one search per primary beam, which increases the work load by a factor of 250,000 for a gain in sensitivity of a factor of 6 . Coherent approaches are being investigated, but this GPU provides a simple solution at minimal cost which will provide the large fraction of the survey goals. Further development of this system could be expected to improve the performance sufficiently for coherent beam searching, perhaps with a limited set of baselines, sky-coverage and parameters.

\section{References}

[1] Camilo etal, 2006, Nature, 442, 892, "Transient pulsed radio emission from a magnetar"

[2] Cordes, 2009, SKA memo 97, "The SKA as a Radio Synoptic Survey Telescope: Widefield Surveys for Transients, Pulsars and ETI"

[3] Deller, etal, 2007, PASP, 119, 853, 318,"DiFX: A Software Correlator for Very Long Baseline Interferometry Using Multiprocessor Computing Environments"

[4] Deneva, etal, 2009, ApJ, 703, 2259, "Arecibo Pulsar Survey Using ALFA: Probing Radio Pulsar Intermittency and Transients"

[5] Dodson etal, CRAFT Survey Proposal, https://pm.atnf.csiro.au/askap/attachments/1758/FASTproposal.pdf

[6] Hankins etal, Nature, 2003, 422, 141, "Nanosecond radio bursts from strong plasma turbulence in the Crab pulsar"

[7] Keith, etal, 2010, MNRAS, subm., "The High Time Resolution Universe Pulsar Survey I: System configuration and initial discoveries"

[8] Lorimer et al, 2007, Science, 318,777, "A Bright Millisecond Radio Burst of Extragalactic Origin"

[9] McLaughlin etal, 2006, Nature, 438, 817, "Transient radio bursts from rotating neutron stars"

[10] Siemion, etal, 2009, AAS, 214, 601.04, "Results from the Allen Telescope Array: The ATA Fly's Eye Transient Search"

We wish to thank W. Van Straten for the Vela data used in Figure 2b. 